\title{
A Novel Power Electronic Load Simulation Method
}

\section{Pengxian Song a , Wenyan Qi b, Haoming Wang c, Zhengzheng Meng d, $\mathrm{Xu} \mathrm{Li}^{\mathrm{e}}$ and Mingzheng Zhu ${ }^{\dagger}$}

\author{
State Grid Tianjin Electric Power Research Institute, Tianjin, 300384, China \\ asongpengxian0821@163.com, bldqiwenyan@126.com, 'cwanghaoming01@126.com, \\ dmzheng123@163.com, e347772054@qq.com, fzmz1220@163.com
}

\begin{abstract}
Keywords: Three-Phase Phase-Controlled Rectifier Bridge, Repetitive Controller, Power Electronic Load.
\end{abstract}

\begin{abstract}
In this paper, a novel power electronic load (PEL) simulation method is proposed. The simulation object is three-phase phase-controlled rectifier bridge load. About this content, there are few articles mentioned. Through the detailed analysis of three- phase phase-controlled rectifier bridge characteristics, a kind of load simulation algorithm is proposed. The algorithm can meet the requirements of simulation in different thyristor trigger angles. A current tracking control strategy of proportional controller and repetitive controller is put forward, which can improve the accuracy of the load simulation. Simulation and experiment verify the accuracy of the load instruction current algorithm, and the effectiveness of the current loop control strategy.
\end{abstract}

\section{Introduction}

Power electronic load (PEL) is a device for testing power quality. It is used to simulate linear loads and nonlinear loads. It is also able to feedback the test energy into the grid [1-3]. The mentioned device topology in this paper is the three-phase back to back converter as shown in Fig. 1. Load simulation converter (LSC) for load simulation is in DBT (Devises Be Test) side and grid connection converter (GCC) for energy-feedback in isolation transformer side. This paper is focused on the LSC. The command current generation algorithm and the current tracking control strategy are the two key technical problems. According to the literature research, the nonlinear load simulation is difficult.

Three-phase uncontrolled rectifier bridge is a typical nonlinear load, [4] [5] studied the algorithm of the single-phase circuit, and whether the method is suitable for three-phase circuits is not discussed. [6] studied the three-phase circuit simulation algorithm, but the diode conduction critical condition description is incomplete and the algorithm is not accurate. As to the current tracking control strategy, the PI or PR controller is used to track the linear current, and the repetitive controller is used to track the nonlinear current in [7] [8].

The non-controlled rectifier bridge is only a special case of phase-controlled rectifier bridge. The phase-controlled rectifier bridge is more able to test the power load capacity. But there is no research on this load simulation algorithm. So this paper will study the simulation method of the three-phase phase-controlled rectifier bridge load. The instruction current algorithm and the current control strategy based on repetitive controller are proposed. The simulation and experimental results verify the validity and accuracy of this method.

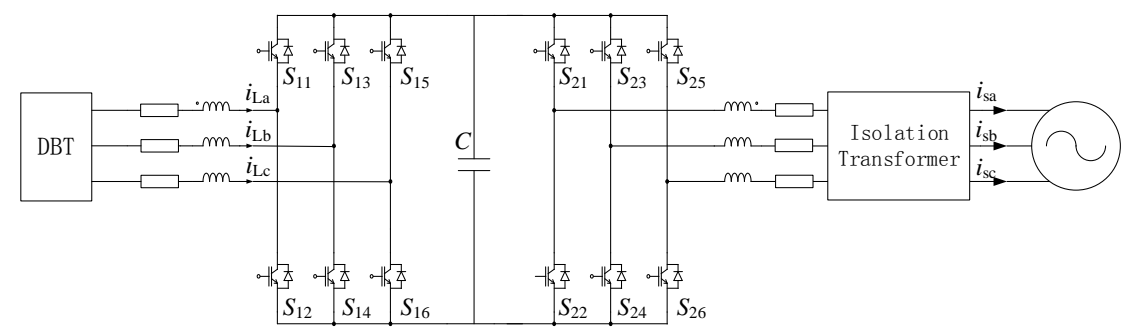

Fig. 1 The circuit of three-phase power electronic load 


\section{The Basic Theory of Load Simulation Algorithm}

The circuit structure of three-phase phase-controlled rectifier bridge is as shown in Fig.2. The AC side equivalent inductance and resistance are neglected. The sequence of thyristor conduction is from one to six. $\mathrm{u}_{\mathrm{R}}$ is the resistance voltage, $\mathrm{u}_{\mathrm{L}}$ is the inductor voltage and $\mathrm{u}_{\mathrm{C}}$ is the capacitor voltage.

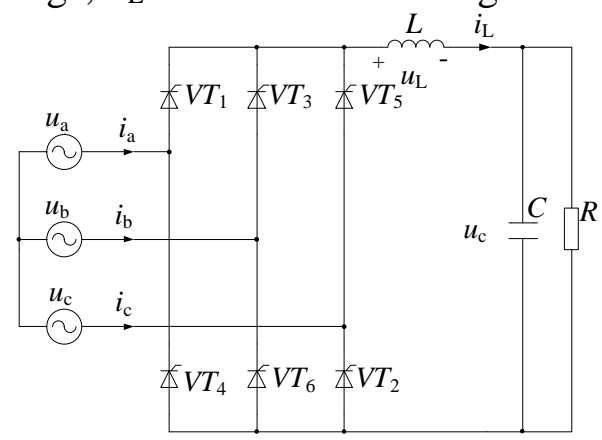

Fig. 2 The circuit of three-phase phase-controlled rectifier bridge

The trigger pulse of $\mathrm{VT}_{1}-\mathrm{VT}_{6}$ is $\mathrm{g}_{1}-\mathrm{g}_{6}$. The pulse trigger sequence is $\mathrm{g}_{1} \rightarrow \mathrm{g}_{2} \rightarrow \mathrm{g}_{3} \rightarrow \mathrm{g}_{4} \rightarrow \mathrm{g}_{5} \rightarrow \mathrm{g}_{6} \rightarrow \mathrm{g}_{1}$. According the working principle of three-phase phased-controlled rectifier bridge, six conduction intervals can be expressed as:

(1): $g_{1} \rightarrow g_{2}, u_{\mathrm{in}}=u_{\mathrm{ab}} ;$ (2): $g_{2} \rightarrow g_{3}, u_{\mathrm{in}}=u_{\mathrm{ac}}$;

(3): $g_{3} \rightarrow g_{4}, u_{\mathrm{in}}=u_{\mathrm{bc}} ;(4): g_{4} \rightarrow g_{5}, u_{\mathrm{in}}=u_{\mathrm{ba}}$;

(5): $g_{5} \rightarrow g_{6}, u_{\mathrm{in}}=u_{\mathrm{ca}} ;$; (6): $g_{6} \rightarrow g_{1}, u_{\mathrm{in}}=u_{\mathrm{cb}}$;

$u_{\text {in }}$ is the AC side voltage corresponding to each conduction interval. Assuming the A-phase voltage is $U_{\mathrm{a}}=U \cos (t+)$, the trigger pulse time of $g_{1}$ is $(t++\pi / 6)$. Because the time difference of the adjacent serial number thyristor conduction is 60 degree, the trigger pulse time of $g_{6}$ is $(t++11 \pi / 6)$. Obviously the interval (1) to (5) are in a fundamental period, while the interval (6)contains the next fundamental period. Assuming the trigger pulse time of $g_{1}$ is $(t++\pi / 3)$, the trigger pulse time of $g_{6}$ is $(t++2 \pi)$. So when the trigger angle is between 0 degree and 30 degree, the circuit equation corresponding to the interval (6) contains two fundamental periods. In a way, when the trigger angle is between 30 degree and 90 degree, the circuit equation corresponding to the interval (5) contains two fundamental periods. When the trigger angle is between 90 degree and 120 degree, the circuit equation corresponding to the interval (4) contains two fundamental periods. In six kinds of conduction interval, writing the corresponding circuit equation can be solved to obtain the AC side current $i_{\mathrm{a}}, i_{\mathrm{b}}$ and $i_{\mathrm{c}}$. According to the similar method in the literature [12], the judging conditions of different circuit equations can be obtained.

The mathematical model is built by PSIM9.0 software platform. Through the comparison of the mathematical model and software model, the accuracy of the simulation algorithm can be verified. The circuit structure is shown in Fig.2 and the main parameter is shown in Tab.1.

Tab.1 The main circuit parameter

\begin{tabular}{|c|c|}
\hline $\operatorname{line} \operatorname{voltage}(u)$ & $100 \mathrm{~V}$ \\
\hline $\operatorname{resistance}(\mathrm{R})$ & $5 \Omega$ \\
\hline inductance(L) $(\mathrm{L})$ & $3 \mathrm{mH}$ \\
\hline capacitance $(\mathrm{C})$ & $500 \mathrm{~F}$ \\
\hline sampling frequence & $10 \mathrm{kHz}$ \\
\hline
\end{tabular}

Since the amplitude of three-phase current is equal and the phase difference is 120 degree, the A-phase current waveform is only given in this paper. The results are shown in Fig.3. The $i_{\mathrm{a}}$ is the simulation result of the PSIM model and the $i_{\mathrm{a}}$ ' is the solving result of the mathematical model. 


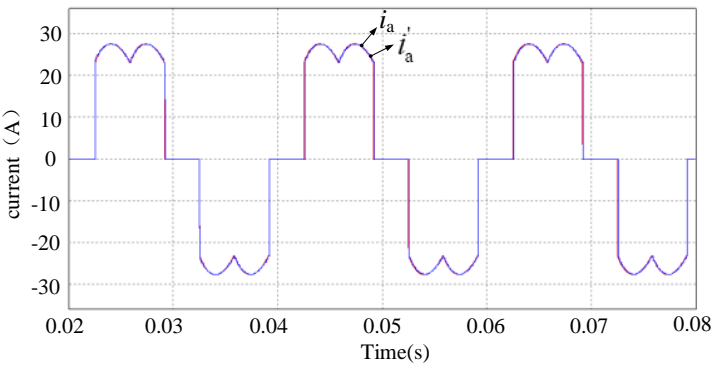

(a) $=15^{\circ}$

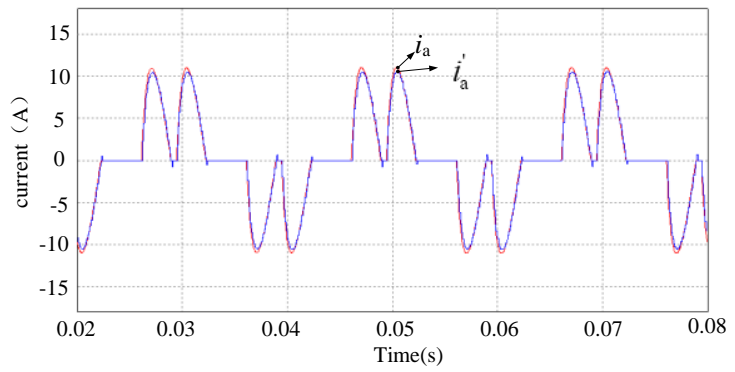

(b) $=95^{\circ}$

Fig. 3 The simulation results of different trigger angle

According Fig.3, we can know that the simulation results of the load simulation algorithm are basically consistent with the results of PSIM model simulation when simulating the steady state characteristics.

\section{The Current Tracking Control Strategy}

The repetitive controller is introduced to expand the current loop bandwidth for realizing the static error tracking. At present, the widely used repetitive controller system block diagram is shown in Fig.4.

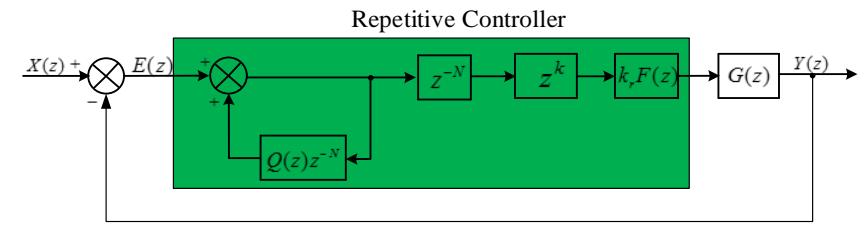

Fig.4 The structure block diagram of repetitive control system

$X(\mathrm{z})$ and $Y(\mathrm{z})$ are the system input and output respectively; $E(\mathrm{z})$ is the error signal; $Q(\mathrm{z})$ is usually taken as a constant less than $1 ; k_{\mathrm{r}}$ is the repetitive controller gain; $z^{\mathrm{k}}$ is the phase compensation link; $z^{-\mathrm{N}}$ is the cycle delay link; $F(\mathrm{z})$ is the dynamic compensator. There is at least one fundamental period delay in the repetitive control and the controller needs to be corrected by the error. So the dynamic response speed is slow. Taking into account the dynamic speed of the PI controller, the control structure of the proportional controller $(\mathrm{P})$ and the repetitive controller is generally used. In order to verify the validity of the control strategy proposed in this paper, the simulation model of three-phase PEL is built by PSIM9.0 simulation software. The main circuit structure is shown in Fig.1. The main circuit parameters are shown in Tab.2. The LSC current tracking control block diagram in coordinate system is shown in Fig.5.

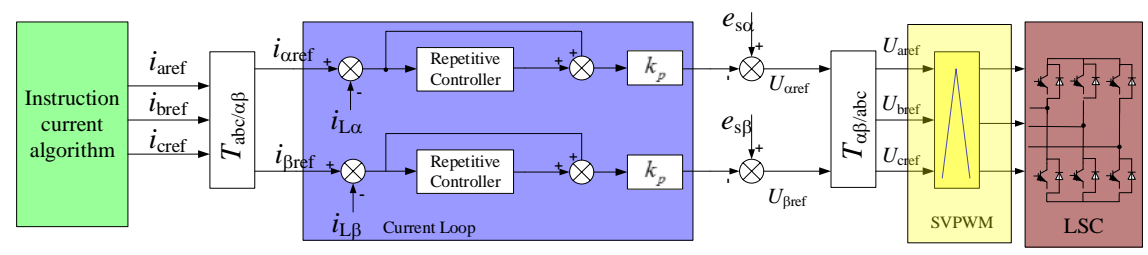

Fig.5 The current tracking control block diagram

Tab. 2 The PEL system paremeters

\begin{tabular}{|c|c|}
\hline grid line voltage $u_{s}$ & $380 \mathrm{~V}$ \\
\hline $\mathrm{DBT} e_{\mathrm{S}}$ & $380 \mathrm{~V}$ \\
\hline$L_{1}, L_{2}$ & $2 \mathrm{mH}$ \\
\hline$r_{1}, r_{2}$ & $0.024 \Omega$ \\
\hline $\mathrm{C}$ & $3000 \mathrm{~F}$ \\
\hline switch frequency & $10 \mathrm{kHz}$ \\
\hline
\end{tabular}

The simulated object is shown in Fig. 2 and the circuit parameters is shown in Tab.2. The trigger angle is 0 degree. In order to test the validity of the composite controller, the simulation results based on PI controller is shown in Fig.6. 


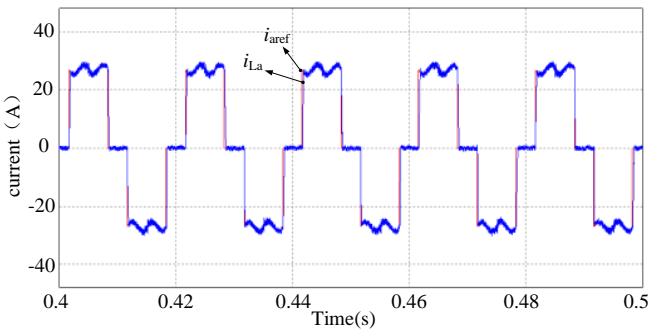

(a) LSC A-phase current and instruction current

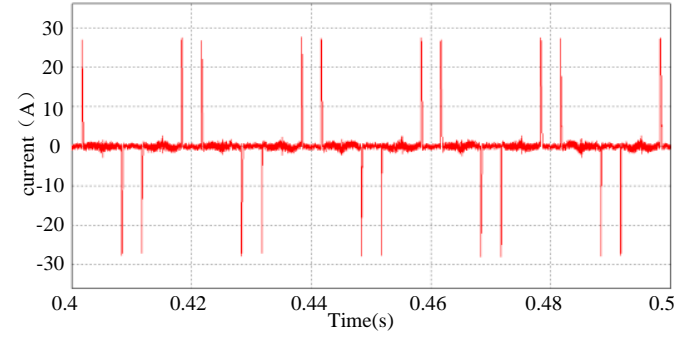

(b) The difference between $i_{\text {aref }}$ and $i_{L a}$

Fig. 6 Simulation waveforms of LSC current based PR controller

Fig.6 (a) is the LSC A-phase current and instruction current; Fig.6 (b) is the difference between $i_{\text {aref }}$ and $i_{\mathrm{La}}$, and the maximum amplitude difference is greater than $20 \mathrm{~A}$.

The simulation results based on the repetitive controller is shown in Fig.7.

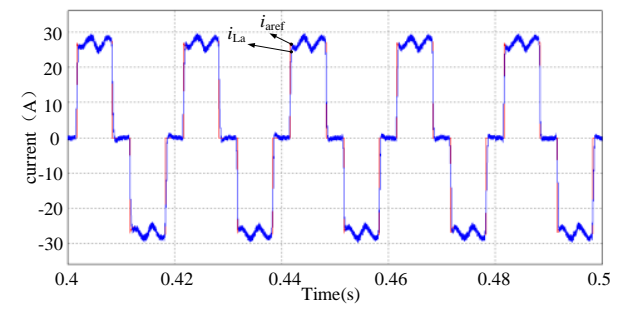

(a) LSC A-phase current and instruction current

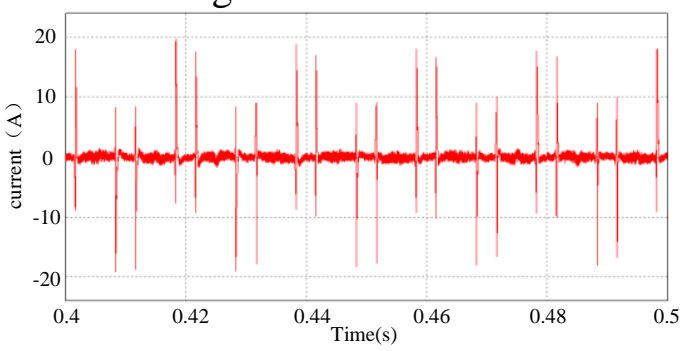

(b) The difference between $\mathrm{i}_{\text {aref }}$ and $\mathrm{i}_{\mathrm{La}}$

Fig.7 Simulation waveforms of LSC current based the compound controller

Fig.7 (a) is the LSC A-phase current and instruction current; Fig.7 (b) is the difference between $i_{\text {aref }}$ and $i_{\mathrm{La}}$, and the maximum amplitude difference is less than $20 \mathrm{~A}$.

From the foregoing, the effect of the current loop tracking using proportional repetitive controller is better than that of the PR controller. The simulation accuracy is higher, and the effectiveness of the proposed control strategy is verified.

\section{Experiment Results}

In order to verify the validity of the proposed control strategy, this paper has carried out the experimental verification on the $380 \mathrm{~V} / 33 \mathrm{kVA}$ prototype platform. Consistent with software simulation parameters, the experimental waveforms can be obtained as shown in the following figures.

The Fig. 8 is the experimental results based on PR controller. Fig. 8 (a) is the LSC three-phase current; Fig. 8 (b) is the LSC A-phase current. The Fig.9 is the experimental results based on the compound controller. Fig.9 (a) is the LSC three-phase current; Fig.9 (b) is the LSC A-phase current.

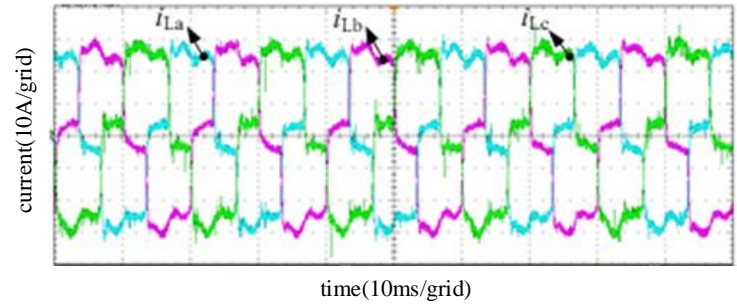

(a) The LSC three-phase current

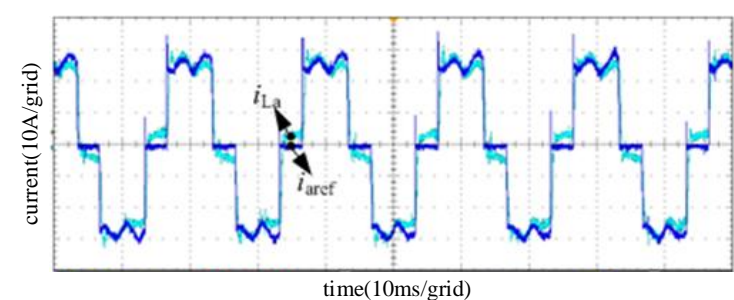

(b) The LSC A-phase current

Fig.8 Experimental waveforms of LSC current based PR controller

As shown in Fig.8, the actual current does not follow the instruction current accurately, and the difference between the two is larger. 


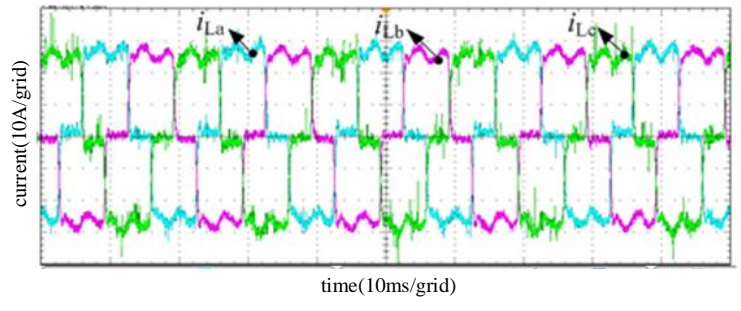

(a) The LSC three-phase current

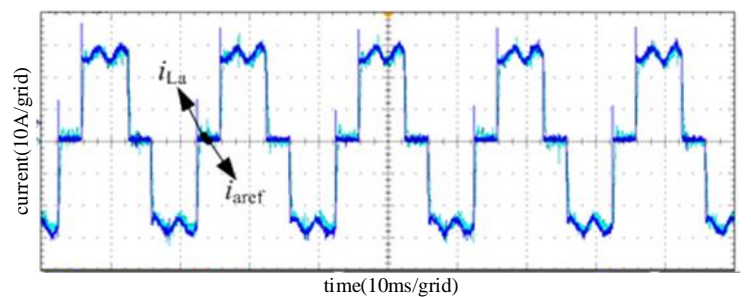

(b) The LSC A-phase current

Fig.9 Experimental waveforms of LSC current based the compound controller

As shown in Fig.9, the actual current can better track the command current, and the relative error of them is relatively small. The experimental results show that the proposed strategy is effective.

\section{Summary}

In this paper, the operating characteristics of the three-phase phase-controlled rectifier bridge are analyzed in detail, and the generation algorithm of the load instruction current is presented. The proposed algorithm increases the load types that can be simulated by PEL.

Considering that the single frequency PR controller can not realize the nonlinear current tracking without static error, a current loop control strategy is proposed in this paper. The simulation and experimental results show that the composite controller of the proportional plus repetitive controller can track the nonlinear command current, and the simulation accuracy is higher than that of the single frequency PR controller.

\section{References}

[1] Tsai M,Tsai C. Energy recycling for electrical AC power sourceburn-in test [J].IEEE Transactions on Industrial Electronics,2000, 47(4).

[2] Huang S J,Pai F S. Design and operation of burn-in test system for three-phase uninterruptible power supplies [J]. IEEE Transactions on Industrial Electronics,2002,49(1).

[3] Zhao Jianfeng, Pan Shifeng, Wang Xun. High power energy feedback AC electroic load and its application in power system dynamic physical simulation [J]. Transactions of China Electotechnical Sociey, 2006, 21(12).

[4]Fen Li. Research of Critical Technology on Single-phase Back-to-back Green Recovery- EnergyBased System for Burn-in Test [D]. Huazhong University of Science \& Technology,2010.

[5] Wang Chengzhi. Research and Design on the Single-phase Power Electronics Load [D]. Huazhong University of Science \& Technology, 2010.

[6] Wu Zhenxing. Research on Characteristics and Design of Three-phase Power Electronics Load [D].Huazhong University of Science \& Technology, 2010.

[7] Huang Zhaoxia, Zou Xudong, Tong Li. Research on Nonlinear Load Simulation of Electric Energy Feedback Load Current Simulator [J]. Transactions of China Electrotechnical Society, 2014, 29(10).

[8] Wang Chengzhi, Zou Xudong, Xu Yun, et al. Improved repetitive control scheme for power electronic load [J]. Proceedings of the CSEE, 2009, 29(12). 